\section{REVIEW ARTICLE}

\section{A. Srinivasan}

S. Mohan

S.K. Mukherji

\title{
Biologic Imaging of Head and Neck Cancer: The Present and the Future
}

\begin{abstract}
SUMMARY: While anatomic imaging (CT and MR imaging) of HNC is focused on diagnosing and/or characterizing the disease, defining its local extent, and evaluating distant spread, accurate assessment of the biologic status of the cancer (cellularity, growth rate, response to nonsurgical chemoradiation therapy, and so forth) can be invaluable for prognostication, planning therapy, and follow-up of lesions after therapy. The combination of anatomic and biologic imaging techniques can thus provide a more comprehensive evaluation of the patient. The purpose of this work was to review the present and future clinical applications of advanced biologic imaging techniques in HNC evaluation and management. As part of the biologic imaging array, we discuss MR spectroscopy, diffusion and perfusion MR imaging, CTP, and FDG-PET scanning and conclude with exciting developments that hold promise in assessment of tumor hypoxia and neoangiogenesis.
\end{abstract}

H NC accounts for approximately 3\%-5\% of all new cancer diagnoses in the United States with 40,000 new cases diagnosed each year. ${ }^{1}$ The present day approach of one-for-all standardized chemoradiation regimens has shown higher locoregional recurrence rates in a sizable proportion of patients, necessitating the development of advanced neuroimaging techniques that can provide biologic information about a cancer and help in identifying cancers potentially unresponsive to standard chemoradiation regimens. If this approach succeeds, these potentially unresponsive cancers can then be treated with other measures, such as increased radiation doses or surgery, which would have a higher chance of achieving success.

When a patient presents with a clinically suspicious head and neck malignancy, the clinician is primarily interested in confirming the suspicion with imaging and in also knowing about the disease extent, locoregional spread, and metastasis. While conventional CT or MR imaging can provide answers to most questions, it falls short in other areas such as detection of metastatic disease in small lymph nodes or prediction of response of a cancer to chemoradiation. Also, when a cancer is treated and the patient presents for follow-up with a mass, it can become difficult in most instances to characterize the lesion by using conventional imaging and typically biopsy is sought to solve the mystery. Hence, the advent of biologic neuroimaging modalities that can look beyond the size and enhancement of a tumor and explore its molecular nature. Knowing about the molecular nature of a tumor, for example

From the Department of Radiology, Division of Neuroradiology, University of Michigan Health System, Ann Arbor, Michigan.

Please address correspondence to Ashok Srinivasan, MD, Division of Neuroradiology, Department of Radiology, University of Michigan Health System, 1500 E Medical Center Dr, Ann Arbor, Ml 48109; e-mail: ashoks@med.umich.edu

Indicates open access to non-subscribers at www.ajnr.org

http://dx.doi.org/10.3174/ajnr.A2535 its cellularity and perfusion, can help identify different biological behavior of tumors (especially with treatment) that would otherwise appear similar on conventional imaging.

In this article, we discuss the current status of advanced neuroimaging techniques pertaining to their application in cancer imaging. In this biologic imaging array, we include proton MR spectroscopy, CTP, perfusion-weighted MR imaging, DWI, and ${ }^{18}$ F-FDG-PET. We also address issues of tumor hypoxia and neoangiogenesis and discuss potential ways of imaging these entities.

\section{Current Clinical Applications in HNC}

In this section, we discuss how each technique can help in the biologic imaging of HNC. Each of these modalities is unique in its ability to detect cancer related to a molecular level and could be considered as a complementary modality. Adding the biologic information obtained from these modalities to the anatomic information can provide a comprehensive assessment of the patient with HNC.

\section{Proton MR Spectroscopy}

Due to the unique ability of MR spectroscopy to detect the presence of specific metabolites in tissues, there has been interest in the development of applications in HNC evaluation. Despite the challenges in obtaining adequate-quality MR spectra in the head and neck due to the small irregular size of lesions and the low signal intensity-to-noise ratios, studies have successfully demonstrated higher concentrations of metabolites in certain pathologies compared with others. ${ }^{2-7}$

Differentiating Normal Tissue and Benign Processes from Malignancy. Elevated Cho/Cr ratios have been demonstrated consistently in HNC compared with normal tissues such as muscle and thyroid glands in both in vivo and in vitro studies, with the ratios increasing with increased TEs. ${ }^{8-11}$ The increased Cho has been attributed to the increased cell mem- 

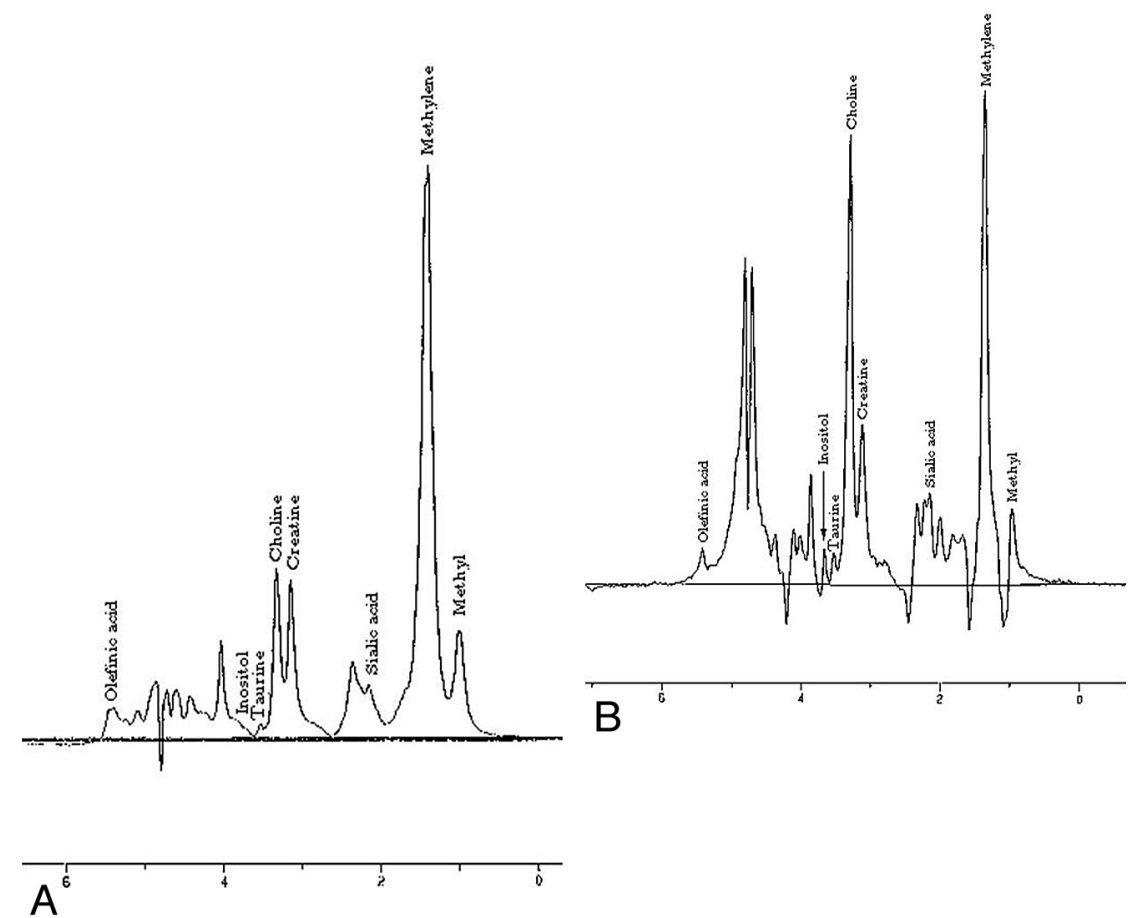

Fig 1. Comparison of MR spectra between muscle $(A)$ and squamous cell carcinoma $(B)$ in the head and neck demonstrates elevation of the Cho peak in squamous cell carcinoma. Analysis of other metabolites like amino acids has also been found to be useful in characterization of HNCs.

brane turnover and metabolic rate in malignancies compared with normal tissues. On the other hand, Cr remains stable even in malignancies because it represents cellular energy stores, which are not altered in neoplasms. Most interesting, a higher likelihood of amino acids being detected in tumor than in normal tissues has also been shown. ${ }^{8,9}$ Figure 1 illustrates the elevation of a Cho peak in HNC compared with normal muscle on a $1.5 \mathrm{~T}$ magnet.

When benign and malignant lesions were studied with MR spectroscopy, higher $\mathrm{Cho/Cr}$ ratios were demonstrated in benign neck tumors (glomus tumors, inverting papilloma, schwannoma) compared with both normal muscle and HNC, with the increased Cho/Cr ratio in benign lesions (compared with malignancies) attributed to the hypercellularity of certain benign neoplasms that show increased cellular proliferation and cell attenuation without harboring true malignancy. ${ }^{12}$ This difference was thought analogous to the presence of an elevated $\mathrm{Cho} / \mathrm{Cr}$ ratio in intracranial meningiomas more than in high-grade gliomas. ${ }^{13,14}$ A later study also showed significant differences in Cho/Cr ratios between benign and malignant salivary gland tumors and also between Warthin tumors and pleomorphic adenomas, suggesting that MR spectroscopy could be used to characterize various salivary gland tumors. ${ }^{15}$

Evaluation of Posttreatment Masses. Because Cho is a marker of cell membrane turnover, residual and recurrent cancers after treatment can be expected to demonstrate this metabolite in excess on MR spectroscopy. This was proved in an MR spectroscopy study of posttreatment masses in which Cho in a mass after treatment correlated significantly with residual cancer $(P=.0046)$. While the absence of Cho in a posttreatment mass had a lesser negative predictive value (81\%; both residual cancers and normal posttherapy tissue could have absent Cho), the presence of Cho in a posttreat- ment mass predicted residual cancer in this study with a $100 \%$ positive predictive value. ${ }^{16}$

Prediction and Monitoring of Response to Therapy. Because MR spectroscopy protocols can vary and are not yet standardized across institutions, variable results have been obtained when using this technique to predict response to therapy in HNC. While an earlier study published in $2005^{17}$ showed that MR spectroscopy had the potential to predict response to therapy (patients with poor tumor response showed elevated Cho/Cr ratios and 1.3:0.9 ppm spectral intensity ratios [signal intensity due to lipid or lactic acid] with $83 \%$ sensitivity and $82 \%$ specificity in predicting treatment failure), a later study has shown that the pretreatment Cho and change in Cho early during a course of treatment did not predict clinical outcome. $^{18}$

To summarize, while MR spectroscopy is a good technique for evaluating abnormal metabolite fractions in lesions and characterizing them as benign or malignant, there is still work to be done in applying it during HNC therapy to monitor or predict response.

\section{CTP}

The basic principle of CTP is the continuous recording of $\mathrm{x}$ ray attenuation over a fixed area of interest during passage of a fast bolus of iodinated contrast medium through the region. This dynamic acquisition covers the first pass of iodinated contrast medium in the regional vascular bed, during which it has an intravascular distribution. ${ }^{19,20}$ This enables creation of time-attenuation curves by using a deconvolution-based algorithm and generation of perfusion parameters such as BV, BF, MTT, and CP, which allow evaluation of the hemodynamic biologic status of a lesion. 

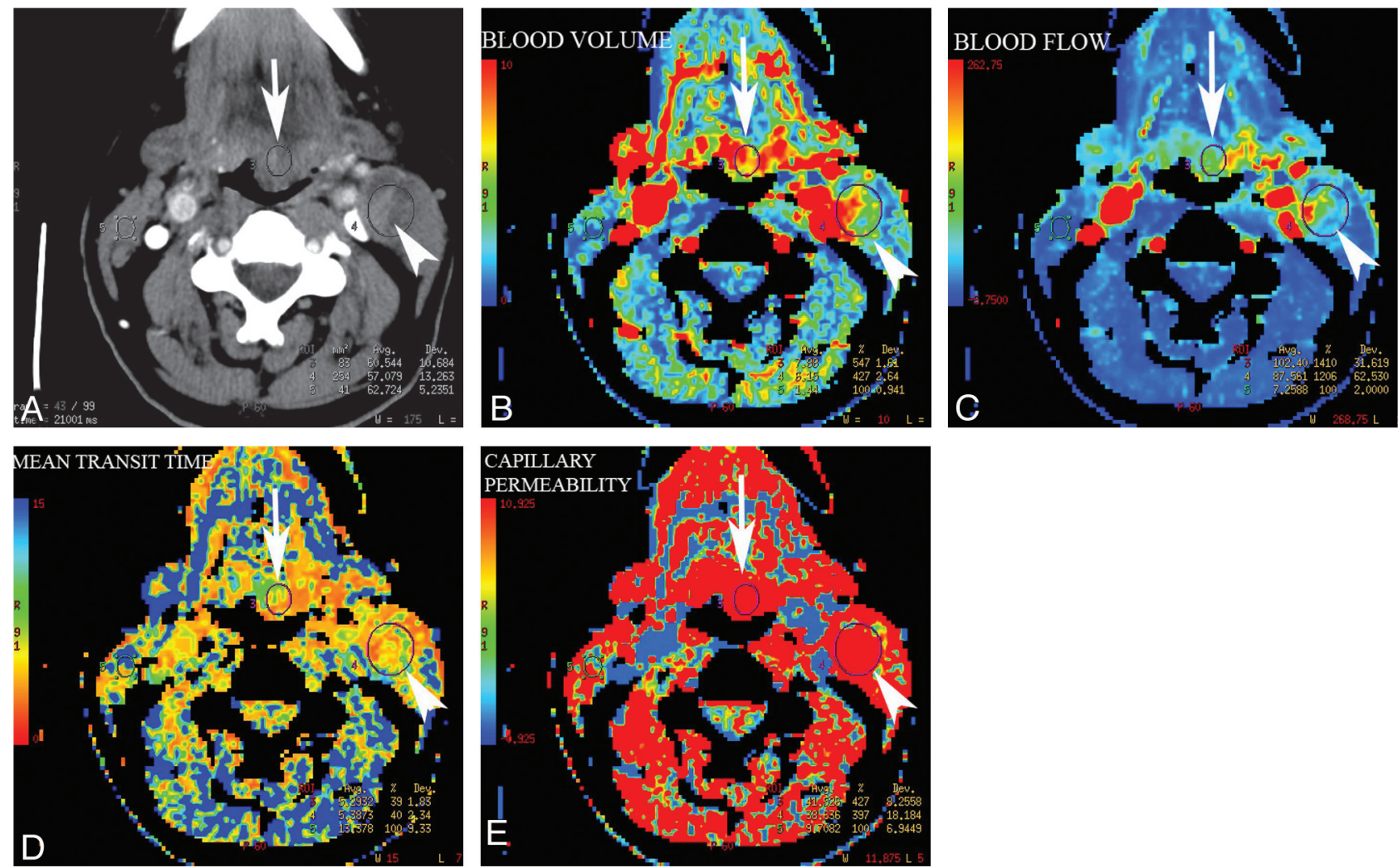

Fig 2. CTP maps generated in a patient with epiglottic cancer and a metastatic necrotic left level II lymph node $(A)$ demonstrate elevated BV $(B)$, elevated $B F(C)$, decreased MTT (D), and increased capillary permeability $(E)$ in both the primary tumor (arrow) and the metastatic node (arrowhead) compared with the normal sternomastoid muscle on the contralateral side.

Differentiation of Malignant from Nonmalignant Lesions. Using CTP to detect the microvascular changes (increased BV or leaky blood vessels) occurring in neoplasms as a result of neoangiogenesis has shown that HNC demonstrates increased $\mathrm{CP}, \mathrm{BF}, \mathrm{BV}$, and decreased MTT compared with normal tissue or benign lesions, with BV sometimes showing overlap among normal salivary glands, benign lesions, and malignant tumors. ${ }^{21,22}$ In 1 study, MTT was thought to be the best discriminator between malignant and nonmalignant pathologies with all lesions with MTT of $<3.5$ seconds being malignant and no malignancies showing MTT of $>5.5 \mathrm{sec}-$ onds. ${ }^{22}$ Figure 2 illustrates the altered perfusion parameters in a patient with squamous cell carcinoma of the epiglottis in both the primary tumor as well as metastatic lymphadenopathy. Both the primary cancer and the level II metastatic lymph node demonstrate elevated BV, elevated BF, decreased MTT, and increased CP.

Differentiating Recurrent Tumors from Posttherapy Changes. Because recurrent tumors can develop neoangiogenesis akin to primary malignancies, CTP is a promising technique to image this neovascularity. A study comparing CTP parameters between recurrent tumors and posttherapy changes has shown that recurrent tumors tend to demonstrate increased BF compared with posttherapy masses (mean BF: 69.71 versus $45.31 \mathrm{~mL} / \mathrm{min} / 100 \mathrm{~g}$ tissue, respectively; $P<.05$ ). However, perfusion parameters could not differentiate benign and malignant lymph nodes, and there was no correlation between perfusion parameters and the tumor volume itself. $^{23}$

Monitoring and Prediction of Response to Therapy. Multiple studies have shown that CTP parameters are helpful in monitoring as well as predicting short-term and long-term response to chemoradiation, though different end points were used in each study to measure response to therapy. While some studies used disease-free survival time, ${ }^{24}$ others relied on FDG-PET scans ${ }^{25}$ or tumor volume reduction ${ }^{26}$ as end points.

Of the parameters studied, BF, BV, MTT, and CP have all proved helpful in predicting response; in some studies, baseline increased $\mathrm{BF}$ and $\mathrm{CP}$ were more predictive of long-term response, ${ }^{24}$ while in others, baseline increased $\mathrm{BV}$ was predictive of short-term response. ${ }^{27}$ Also, during the course of chemoradiation, responders showed a significant reduction in $\mathrm{BF}$ and $\mathrm{BV}$, whereas nonresponders showed a nonsignificant elevation in $\mathrm{BF}, \mathrm{BV}$, and $\mathrm{CP} .^{27}$

Noninvasive Measurement of Intratumoral MVD. Intratumoral MVD is a marker for tumor angiogenesis, with increased MVD considered an increased risk of local-regional recurrence, distant metastasis, and decreased survival in patients with various cancers, including breast, lung, cervix, esophagus, melanoma, and prostate. ${ }^{28}$ Hence measurement of MVD in HNC can be useful for prognostication. CT has proved useful in this regard, with BV measured in HNC showing positive correlation with MVD, implying that increased BV represents increased MVD. ${ }^{28}$

In summary, CTP can provide noninvasive measures of tumor microvascularity in HNC, which can be key for identification of abnormal tissues in the posttherapy setting and prediction and monitoring of response to nonsurgical therapies. CTP is probably a more reproducible technology across institutions, especially with the widespread presence of multi- 
detector CT, thus making it one of the potential workhorses of biologic imaging of HNC.

\section{Perfusion-Weighted MR Imaging}

Akin to CTP, perfusion-weighted MR imaging also measures $\mathrm{BF}$ dynamics at the microcirculation level and is able to generate parameters such as BV, BF, and MTT. There are several techniques for performing perfusion-weighted MR imaging, of which the most widely available is $\mathrm{T}^{\star}$-weighted DCE imaging. This requires the bolus intravenous administration of a paramagnetic contrast medium and the rapid acquisition of images with time during the first pass of contrast material through the capillary bed. ${ }^{29}$ Other perfusion-weighted MR imaging techniques include dynamic T1-weighted contrast imaging (providing assessment of tumor permeability) and arterial spin-labeling (a perfusion technique that uses intraarterial water as an endogenous tracer and hence does not require administration of intravenous gadolinium). While these latter techniques are promising, they are less widely available in the clinical arena and their use is presently limited to a few centers. ${ }^{30}$

Differentiation of Tumor and Normal Tissues. In 2008, a study using first-pass DCE perfusion-weighted MR imaging in both benign and malignant head and neck tumors was able to demonstrate the feasibility of the technique in quantifying perfusion parameters. The authors also showed that all perfusion parameters (including BF, BV, extravascular extracellular volume, difference in bolus arrival time between arterial input and tissue, MTT, permeability, and extraction ratio maps) were significantly different between tumor and normal muscle tissue ${ }^{31}$ thus suggesting that perfusion-weighted MR imaging had the potential to be an additional tool in biologic imaging of HNC.

Monitoring and Prediction of Response to Therapy. In a prospective study of DCE T1-weighted perfusion-weighted MR imaging in HNC, the authors were able to show that there was significantly increased BV (in the primary gross tumor volume) after 2 weeks of chemoradiation in patients who had local disease control (at a median 10 months) compared with those who had local or regional failure. ${ }^{32}$ Most surprising, reduction in tumor volume after 2 weeks of chemoradiation did not predict local disease control in this study. Figure $3 \mathrm{dem}-$ onstrates the transient increase in BV (during midtherapy) that occurred in 1 of the patients who showed complete response to therapy. While this preliminary work suggested that perfusion-weighted MR imaging may also have a role in monitoring therapy response, a more recent study investigating the efficacy of pretreatment DCE perfusion-weighted MR imaging parameters in predicting response has shown that the pretreatment $K^{\text {trans }}$ value was significantly higher $(P=.001)$ in complete responders compared with partial responders $\left(0.64 \pm 0.11\right.$ minutes $^{-1}$ versus $0.21 \pm 0.05$ minutes $^{-1}$, respectively). $K^{\text {trans }}$ is thought to be a measure of the permeability surface area product per unit volume of tissue for endothelial transport between plasma and extracellular extravascular space and is, therefore, a key measurement in tumor neoangiogenesis.

Thus, it appears that perfusion MR imaging could be a useful technique for noninvasive measurement of tumor perfusion, but there are still issues with optimization of protocols
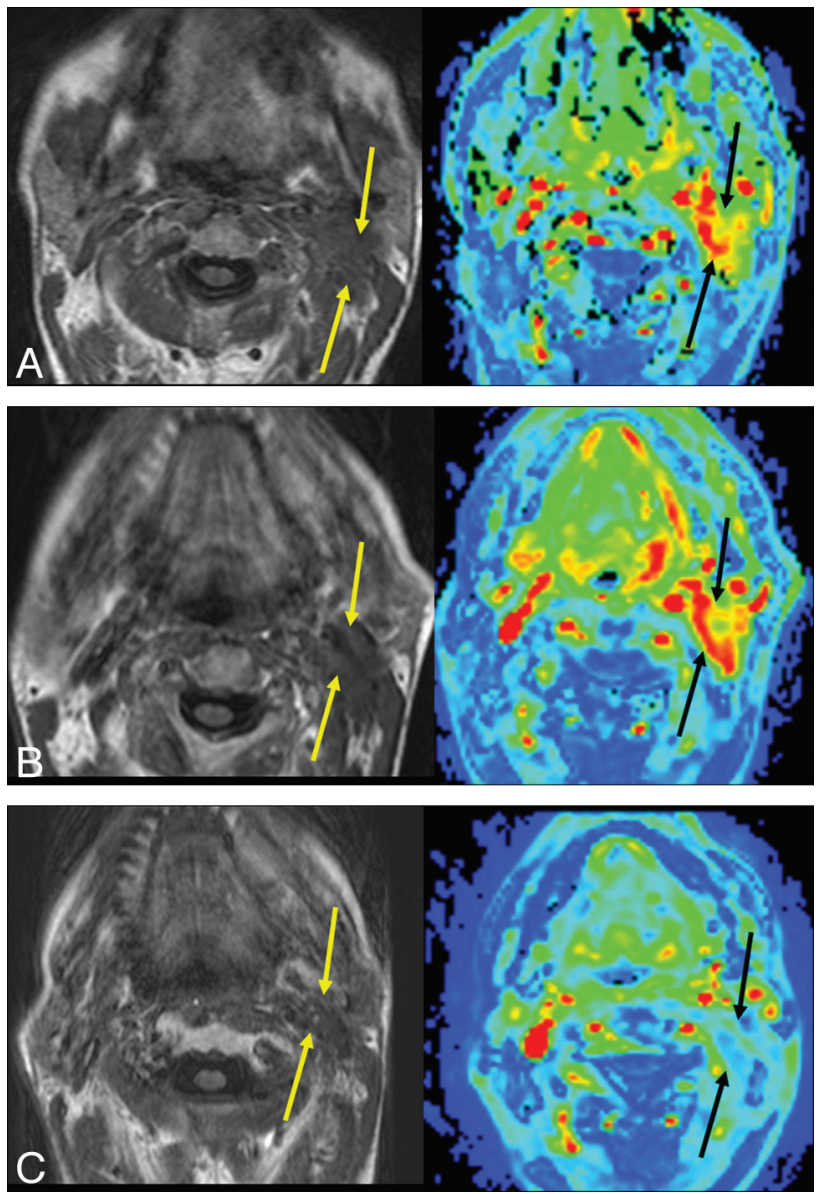

Fig 3. $A$ and $B$, Axial T1-weighted and BV dynamic contrast-enhanced MR perfusion images in a patient with left neck squamous cell carcinoma demonstrate relatively increased BV $(A)$ on the pretherapy scan, further increase $(B)$ during midtherapy (3 weeks during radiation therapy), and eventual decreases $(C)$ at the end of therapy (6 weeks after completion). The patient had a complete response of tumor to therapy as seen on follow-up CT-PET imaging.

and longer scanning times compared with CTP that need to be addressed before it can become prime time.

\section{DWI}

Due to its ability to reflect water molecular motion in the tissue microenvironment, DWI has found various applications in head and neck imaging. The basic premise behind DWI in the head and neck is that tissues that are more compact at a molecular level (eg, tissues with higher cellularity) tend to show a relative reduction of water molecular motion expressed as lower ADC values.

Differentiation of Benign and Malignant Processes. It is generally agreed that malignant lesions in the head and neck tend to demonstrate lower ADC values than benign processes or normal tissues, likely due to their hypercellular nature. ${ }^{33-38}$ Most studies have shown that primary malignancies in the head and neck and metastatic nodes show ADC values below $0.9-1.3\left(\times 10^{-3} \mathrm{~mm}^{2} / \mathrm{s}\right)$; the optimal threshold for differentiation is still an unknown. The utility of ADC is the additional confidence of calling a lesion "probably benign," thereby avoiding unnecessary biopsy. Figure 4 illustrates the MR imaging features (including an ADC map) in a 57-year-old patient with a right parotid lesion considered indeterminate on clinical examination. The decreased ADC value $\left(0.71 \times 10^{-3}\right.$ 

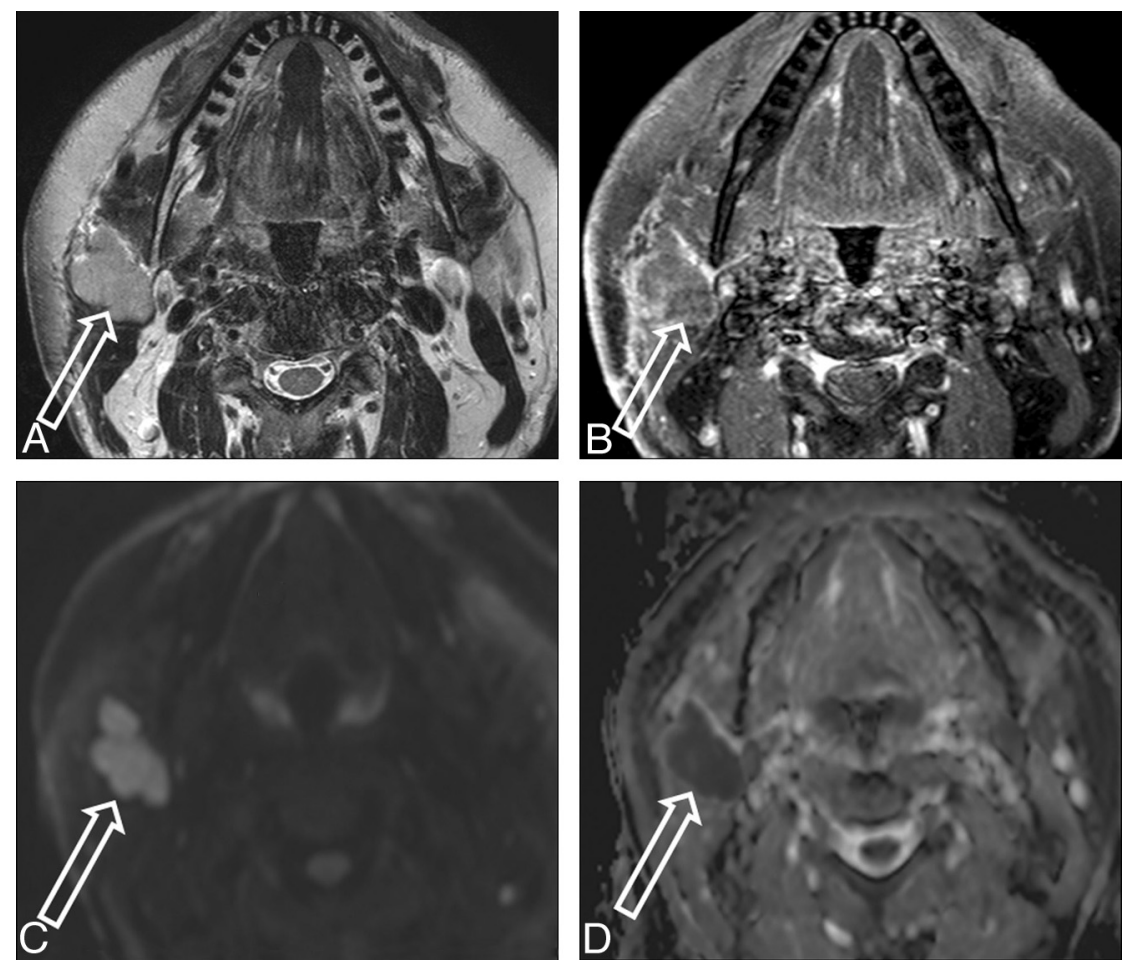

Fig 4. Axial T2-weighted $(A)$ and postcontrast $\mathrm{T} 1$-weighted $(B)$ images in a 57-year-old patient demonstrate a T2 hyperintense, heterogeneously enhancing lesion arising from the right parotid gland. The lesion is bright on the $b=800$ diffusion image $(C)$ and shows restriction of diffusion on the ADC map (D), with the ADC measuring $0.712 \times 10^{-3} \mathrm{~mm}^{2} / \mathrm{s}$. This was resected with a final pathologic diagnosis of neuroendocrine carcinoma.

$\mathrm{mm}^{2} / \mathrm{s}$ ) and the peripheral heterogeneous enhancement increased the suspicion that the lesion was probably malignant. Biopsy revealed neuroendocrine cancer.

Salivary Gland Tumors and Xerostomia Evaluation. While some studies have shown that ADC can help distinguish benign and malignant parotid tumors, others have shown no difference in ADC between parotid tumors and normal tissue or between HNC and normal parotid glands. ${ }^{34,37,39-41}$ In the setting of postradiotherapy xerostomia, DWI can prove helpful by demonstrating serial ADC changes suggestive of reduced glandular function. The normal parotid gland shows a decrease in ADC during early gustatory stimulation, followed by a steady increase in ADC that becomes significantly higher than the baseline. Loss of these serial ADC changes can be suggestive of glandular involvement by radiation therapy - induced xerostomia, thus providing a noninvasive method of assessing glandular function. ${ }^{42}$

Tumor Recurrence. Identification of recurrent tumor in the posttherapy setting is often challenging; the dilemma between calling a posttherapy mass a recurrent tumor or posttherapeutic benign changes can be lessened to some extent by evaluating the DWI. Recurrent tumors show a decreased ADC compared with nonmalignant changes or radionecrosis, presumably due to increased free water in necrosis and increased cellularity in recurrent tumors. ${ }^{43-45}$ While there is no single $\mathrm{ADC}$ value or a range of ADC values that can diagnose recurrent tumor with certainty, these values can increase the confidence that the lesion is probably not recurrent tumor. Figure 5 illustrates 1 such scenario in which ADC values in the posttherapy setting could make a distinction between recurrent tumor and benign granulation tissue.
Prediction and Monitoring of Response to Nonsurgical Therapy. On the basis of the hypothesis that tumors that respond to chemoradiation would undergo greater treatmentinduced loss of tumor cells (and an increase in free water), resulting in increased $\mathrm{ADC}$ values, an earlier study in a mouse model of HNC was able to show that an increase in ADC during treatment correlated with improved survival. ${ }^{46}$ Use of a novel technique (PRM [ADC] that measures voxel-to-voxel changes in ADC) in 15 patients with HNC has also demonstrated that changes in PRM (ADC) and percentage change in tumor volumes between baseline DWI and 3-weeks' midtherapy DWI showed correlation with outcomes, as opposed to percentage change in mean ADC, which did not correlate with tumor control at 6 months. ${ }^{47}$ Results from these studies imply that ADC values may be more accurate in detecting changes in tumor architecture, possibly at an earlier stage, compared with conventional measures such as alterations in tumor volume or enhancement.

Both baseline ADC and change in ADC during therapy have proved useful as predictors of response in a recent study in which the authors showed that tumors that responded completely to chemoradiation had decreased baseline ADC values compared with tumors that responded only partially; in this study, change in the ADC during therapy served as a better predictor than baseline ADC alone. ${ }^{48}$ Other studies have also corroborated these findings by demonstrating that not only do baseline tumor ADC values correlate inversely with tumor regression rates after therapy but they also show good correlation between changes in ADC occurring during early therapy and 2-year locoregional recurrence. ${ }^{49,50}$ While it appears from these studies that more cellular tumors (with decreased base- 

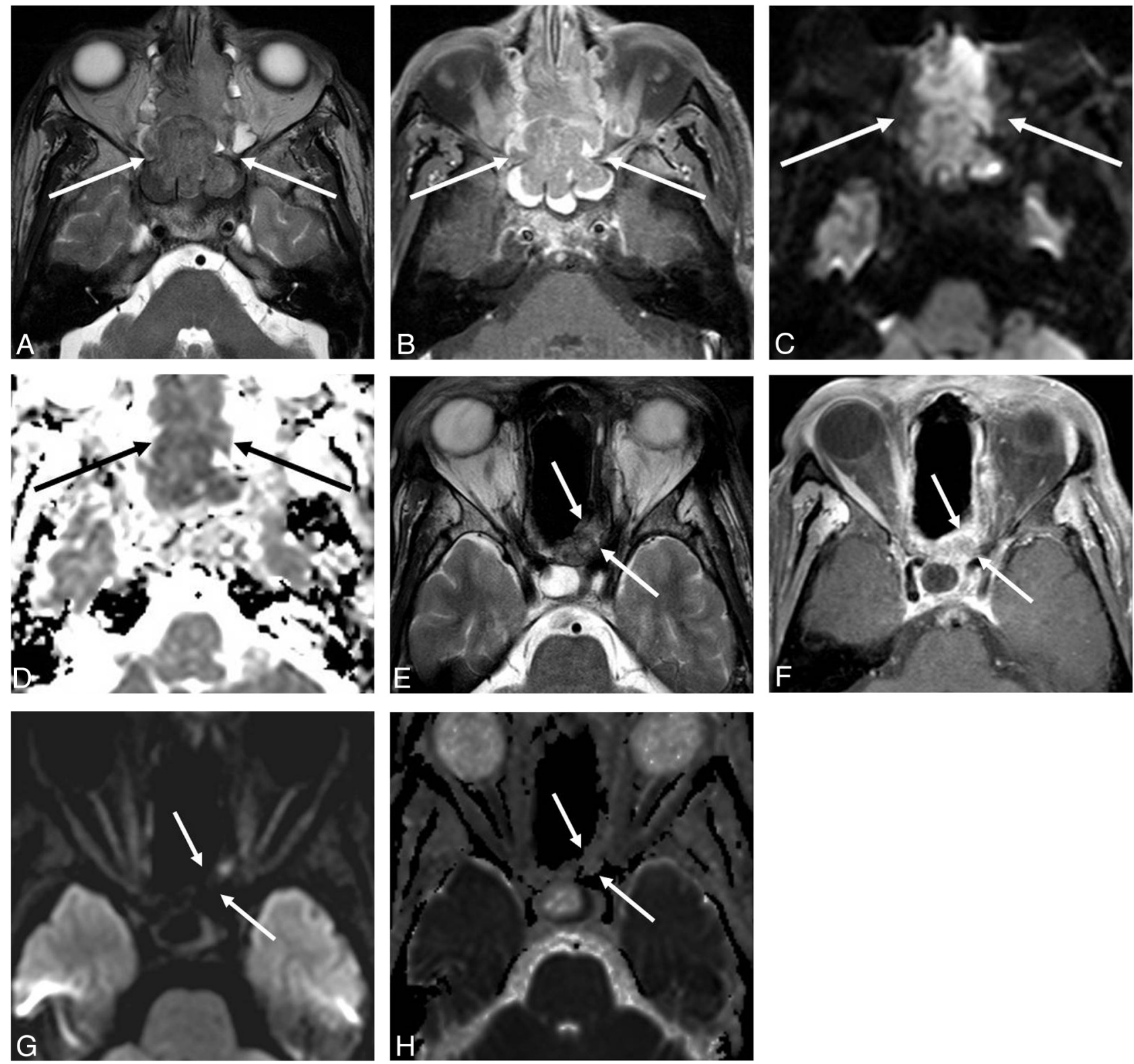

Fig 5. Pre- and postsurgical MR images in a patient with sinonasal undifferentiated carcinoma within the nasal cavity and ethmoid sinuses. $A-D$, Presurgical images demonstrate a T2 hypointense $(A)$ enhancing lesion $(B)$ with increased diffusion signal intensity on the $b=800$ image $(C)$ and restriction of diffusion $\left(\operatorname{ADC}=0.701 \times 10^{-3} \mathrm{~mm}^{2} / \mathrm{s}\right)$ on the $A D C \mathrm{map}(D)$. Six months after surgery and radiation therapy, the patient presented with epistaxis, prompting re-imaging. $E$ and $F$, MR imaging shows a small T2 hypointense focus on the left side $(E)$ with intense enhancement (F). $G$ and $H$, However, the focus is only mildly hyperintense on the $b=800$ diffusion image $(G)$ and is not restricting diffusion $\left(A D C=1.8 \times 10^{-3} \mathrm{~mm}^{2} / \mathrm{s}\right)$ on the ADC map $(H)$, suggesting that this is probably not tumor recurrence. However, biopsy was still performed and pathology revealed benign granulation tissue. With further support from the literature, it may be possible to avoid biopsies in patients demonstrating ADC values not consistent with tumor recurrence.

line ADC) may respond better to chemoradiation, a more recent study has shown that only the posttreatment ADC was a marker for locoregional failure and not pre- or intratreatment ADC measurements. Therefore, the true value of ADC in prediction and monitoring of response likely lies in a combination of pre-, intra-, and posttherapy ADC values, which needs to be worked out in larger samples to establish its utility.

Thus DWI has been one of the most investigated biologic imaging modalities in HNC, primarily due to its ability to acquire reproducible images in a short sequence that can be built into any head and neck MR imaging protocol. Unfortunately, there has been the issue of magnetic-susceptibility artifacts, especially at the level of dental fillings, which has precluded its widespread application in all types of HNC. As one of the potential solutions, parallel imaging can be used in these instances to reduce susceptibility artifacts and acquisition times.

\section{FDG-PET}

In contrast to the other modalities discussed so far, FDG-PET imaging relies on transport of radiolabeled glucose into the cell, thus providing a direct way of visualizing abnormal cells. This principle has been extensively used in the diagnosis and follow-up not only of HNC but also of cancers in various other body parts. Avidity for FDG is not restricted to neoplastic processes and can be seen with inflammatory lesions as well.

Evaluation for Residual or Recurrent Disease. FDG$\mathrm{PET} / \mathrm{CT}$ is one of the most accurate noninvasive modalities presently available for differentiating posttreatment changes 

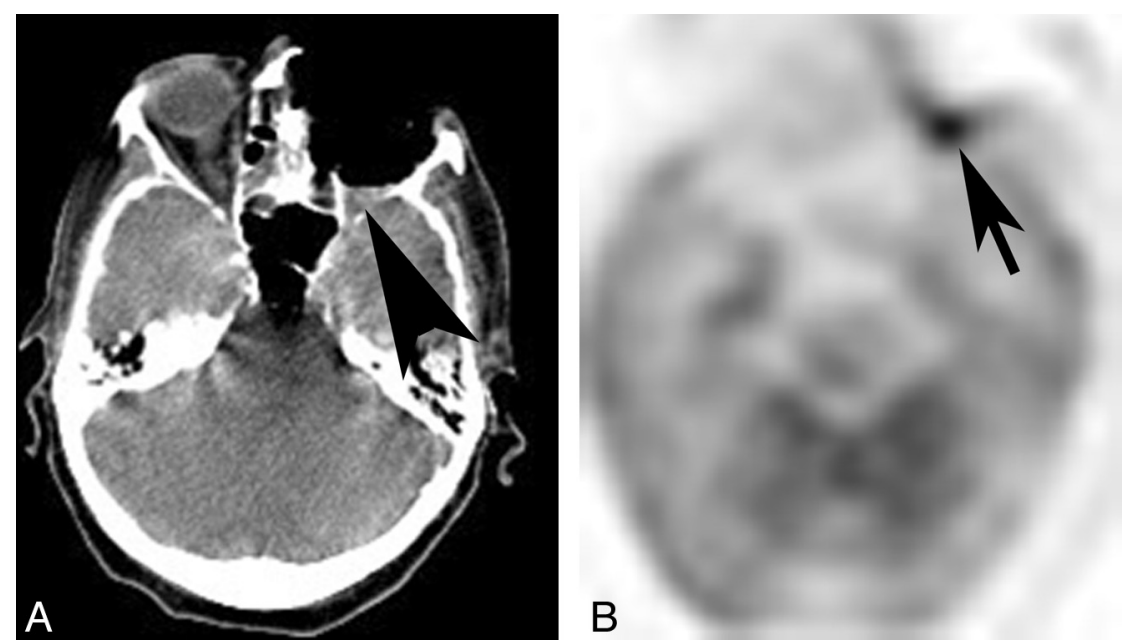

Fig 6. A, CT scan obtained as part of PET/CT fusion imaging in a patient with orbital squamous cell carcinoma treated with exenteration shows soft tissue at the orbital apex (arrowhead), which did not show any enhancement with contrast administration (not shown). B, Corresponding FDG-PET image shows intense FDG avidity, raising the suspicion for tumor recurrence, which was confirmed on pathology. FDG-PET imaging can be helpful for detection of residual and recurrent malignancies in the head and neck.

from residual or recurrent disease. ${ }^{51,52}$ A whole-body FDG$\mathrm{PET} / \mathrm{CT}$ is not only helpful in excluding residual disease and identifying candidates for salvage surgery after chemoradiation but also allows imaging of the primary site, neck, and distant metastases in 1 imaging study. ${ }^{53}$ While there may be false-positives (brown fat, asymmetric muscular activity, inflammation from infection or previous therapy) or false-negatives (small tumor size, low glycolytic activity), the specificity and negative predictive value of FDG-PET/CT has been consistently high ( $89 \%$ and $97 \%$, respectively) for the detection of residual neck disease in patients with $\mathrm{HNC}$ who were treated with concurrent chemoradiation. ${ }^{54}$ Also, it has been shown that the fraction of false-positive studies could be reduced from $27 \%$ to $10 \%$ by combined PET/CT rather than by structural imaging alone, while maintaining a high negative predictive value of $98 \%$. $^{54}$

FDG-PET has a high sensitivity and moderate specificity for detecting recurrent disease at the primary tumor site, regional lymph node metastases, and distant metastases. ${ }^{53}$ Meta-analysis has shown that the pooled sensitivity, specificity, and positive and negative predictive values of FDG-PET for detecting residual or recurrent HNC were 94\%, 82\%, 75\%, and 95\%, respectively. ${ }^{55}$ Figure 6 demonstrates tumor recurrence in the left orbital apex seen as an FDG-avid focus; CT imaging alone could not make the distinction between recurrent tumor and granulation tissue.

Treatment-Response Assessment. FDG-PET/CT can be used to assess treatment response in HNC, with most studies indicating that the efficacy of chemoradiation may not be assessed fully for at least 8-10 weeks after completion due to radiation-induced inflammation. It is generally accepted that FDG-PET be performed 10-12 weeks after treatment completion to reduce the false-positives. ${ }^{55}$ In contrast, CTP, DWI, and perfusion-weighted MR imaging data have shown that these other modalities may be capable of monitoring and predicting response at a much earlier stage during therapy. ${ }^{24,27,32,47-50}$

Obtaining Prognostic Information. A recent study showed that negative findings on FDG-PET/CT at 6 months after the completion of radiation therapy were associated with significantly improved 2-year locoregional control (95\% versus $46 \%$ ), distant control (95\% versus $46 \%$ ), progression-free survival (93\% versus $30 \%$ ), and overall survival (100\% versus $32 \%$ ) compared with patients with positive findings on FDGPET/CT studies, suggesting a role for PET imaging in longterm prognostication of HNC. ${ }^{56}$

Evaluation of Tumor Volume for Radiation Therapy Planning. FDG-PET/CT is useful in aiding GTV contouring because the metabolic activity of the tumor demarcates the boundary between active tumor and normal surrounding tumor. ${ }^{57}$ FDG-PET may also reduce interobserver variability in GTV delineation, reduce the size of the GTV, identify tumor areas or lymph nodes missed by CT or MR imaging, and identify parts of the GTV potentially requiring an additional radiation dose. ${ }^{58}$ While all modalities may overestimate the extent of tumor (FDGPET by $29 \%$, CT by $65 \%$, and MR imaging by $89 \%$ ), a reduction in GTV by using 18F-FDG-PET has been demonstrated in laryngeal cancer, with FDG-PET coming closest to depicting the true tumor volume. ${ }^{58}$ The 2-year overall survival and event-free survival rates of patients treated with FDG-PET/CT-based intensity-modulated radiation therapy were $91 \%$ and $80 \%$, respectively, and were significantly better than those in the control group who received standard $3 \mathrm{D}$ conformal radiation therapy. ${ }^{58}$

Thus, FDG-PET continues to be the workhorse of posttherapy imaging for the assessment of residual or recurrent disease, with other biologic modalities starting to play an important role in early monitoring and prediction of response to chemoradiation. While it is exciting to image at a biologic level and provide information about the potential response of an HNC to therapy, there are other variables that likely affect treatment response, such as tumor hypoxia. In the next few sections, we will present emerging cutting-edge applications that can propel biologic imaging of HNC into the realm of the standard of care.

\section{Emerging Applications}

\section{DTI}

DTI is presently in its infancy in the development of applications in the head and neck. A feasibility study performed in 2009 on a 3T magnet has demonstrated that it was possible to 
define the relationship between the inferior alveolar nerves and adjacent tissues by using DTI and tractography. ${ }^{59}$ This information can be paramount to salvaging important neural bundles during surgical removal of masses, which would require accurate identification of the nerves themselves.

\section{Imaging of Hypoxia}

Hypoxia represents a negative prognostic factor for radiation treatment of HNC and is associated with significant resistance to chemoradiation. ${ }^{53}$ Hypoxic cells are resistant to the cytotoxic effects of chemotherapy and ionizing radiation and require radiation doses up to 3 times higher than those in the same cells under normoxic condition to achieve the same level of cell inactivation. ${ }^{60}$ Hence, there is growing interest in diagnosing hypoxic HNC before therapy in the hope of applying novel treatment strategies that may overcome resistance to conventional chemoradiation. ${ }^{60}$

Potential tumor hypoxia imaging agents include ${ }^{18} \mathrm{~F}$ FMISO and copper 60 (II)-diacetyl-bis(N4-methylthiosemicarbazone). ${ }^{18} \mathrm{~F}$-FMISO is reduced and bound to cell constituents under hypoxic conditions with the level of hypoxia depicted by ${ }^{18} \mathrm{~F}$-FMISO before treatment, correlating with locoregional failure and the absence of hypoxia associated with a low risk of locoregional failure. ${ }^{58,61,62}$ Treating patients with hypoxic primary tumors with additional cytotoxin resulted in significantly fewer local failures than treating patients with chemotherapy alone, thus showing that hypoxia identification by using biologic imaging can be crucial for prognostication. ${ }^{62}$

\section{Imaging of Tumor Cell Proliferation}

Because tumor cell proliferation during the course of therapy adversely affects radiation-treatment outcome and prognosis in HNC, a novel PET agent was developed that reflects the activity of thymidine kinase 1 , a principal enzyme in the salvage pathway of DNA synthesis. ${ }^{63,64}$ The $3^{\prime}$-deoxy- $3^{\prime}-{ }^{18} \mathrm{~F}$ FLT-PET signal intensity is more specific for actively dividing tumor cells than the ${ }^{18}$ F-FDG-PET signal intensity. During the course of chemoradiation, there is a significant inflammatory response that can result in spurious ${ }^{18} \mathrm{~F}$-FDG-PET signal intensity; thus, the use of a proliferation-specific PET tracer, such as ${ }^{18} \mathrm{~F}$-FLT, may prove helpful because its uptake depends on the proliferative activity of the primary tumor and is not affected by the surrounding inflammatory tissue. ${ }^{63-65}$ In a prospective study of patients with suspected recurrent and primary laryngeal cancer, both ${ }^{18} \mathrm{~F}$-FLT and ${ }^{18} \mathrm{~F}$-FDG-PET were able to detect pathologically proved cancer in 15 of 17 patients. ${ }^{65}$ Another study using both ${ }^{18}$ F-FLT and ${ }^{18}$ F-FDG-PET in untreated patients with HNC demonstrated significant inverse correlation between both PET tracers and survival but no correlation between ${ }^{18}$ F-FLT uptake and Ki-67 (proliferative molecular marker) expression level. ${ }^{66}$ The authors concluded that ${ }^{18} \mathrm{~F}$-FLT does not provide additional visual information in comparison with ${ }^{18} \mathrm{~F}$-FDG. Hence it appears that more research is needed before ${ }^{18} \mathrm{~F}$-FLT-PET can prove more helpful than ${ }^{18} \mathrm{~F}$-FDG-PET alone.

\section{Inhibition of Neoangiogenesis}

${ }^{18} \mathrm{~F}$-Galacto-RGD-PET imaging of avB3 expression, a receptor related to tumor angiogenesis and metastasis, may be used in planning and response evaluation of avB3-targeted thera- pies. ${ }^{53}$ The intensity of ${ }^{18} \mathrm{~F}-\mathrm{RGD}$ peptide accumulation correlates with the presence of activated endothelial cells and, quantitatively, with microvessel attenuation. ${ }^{60}$

\section{PET/MR Imaging}

Combined PET/MR imaging in HNC shows promise in increasing accuracy. A recent study showed that the accuracy for tumor-node-metastasis staging was not different between PET/CT and MR imaging (74.6\% and 73\%, respectively). When the MR imaging information was combined with that of $\mathrm{PET} / \mathrm{CT}$, the accuracy improved to $92 \%$. These findings suggest that the emerging PET/MR imaging technology will play an important role for managing patients with HNC. ${ }^{67}$ PET/MR imaging may further improve the accuracy of GTV delineation in oropharyngeal and oral cavity tumors. ${ }^{58}$

\section{Summary}

While the traditional role of imaging has remained in the anatomic domain, the recent developments in technology have allowed evolution of imaging modalities that can assess the biologic domain of HNC, thus helping in better understanding of cancer pathophysiology and allowing better evaluation of treatment strategies. In summary, biologic imaging of HNC has come out of the shadows of the research lab and is becoming more applicable in the daily evaluation of patients. The information that is and can be obtained will help propel us toward customization of treatments and better prognostication, eventually altering the landscape of HNC care.

Disclosures: Suresh Mukherji, Consultant. Philips, Bayer, GE, Details: consulted during the past year.

\section{References}

1. American Cancer Society. www.cancer.org. Accessed October 28, 2007

2. Delikatny EJ, Russell P, Hunter JC, et al. Proton MR and human cervical neoplasia: ex vivo spectroscopy allows distinction of invasive carcinoma of the cervix from carcinoma in situ and other preinvasive lesions. Radiology 1993; 188:791-96

3. Gill SS, Thomas DG, Van Bruggen N, et al. Proton MR spectroscopy of intracranial tumours: in vivo and in vitro studies. J Comput Assist Tomogr 1990;14:497-504

4. Negendank WG, Brown TR, Evelhoch JL, et al. Proceedings of a National Cancer Institute workshop: MR spectroscopy and tumor cell biology. Radiology 1992;185:875-83

5. Shah GV, Gandhi D, Mukherji SK. Magnetic resonance spectroscopy of head and neck neoplasms. Top Magn Reson Imaging 2004;15:87-94

6. Vogl T, Peer F, Schedel H, et al. 31P-spectroscopy of head and neck tumors: surface coil technique. Magn Reson Imaging 1989;7:425-35

7. Yeung DK, Fong KY, Chan QC, et al. Chemical shift imaging in the head and neck at 3T: initial results. J Magn Reson Imaging 2010;32:1248-54

8. Mukherji SK, Schiro S, Castillo M, et al. Proton MR spectroscopy of squamous cell carcinoma of the extracranial head and neck: in vitro and in vivo studies. AJNR Am J Neuroradiol 1997;18:1057-72

9. Mukherji SK, Schiro S, Castillo M, et al. Proton MR spectroscopy of squamous cell carcinoma of the upper aerodigestive tract: in vitro characteristics. AJNR Am J Neuroradiol 1996;17:1485-90

10. King AD, Yeung DK, Ahuja AT, et al. In vivo proton MR spectroscopy of primary and nodal nasopharyngeal carcinoma. AJNR Am J Neuroradiol 2004;25:484-90

11. King AD, Yeung DK, Ahuja AT, et al. In vivo $1 \mathrm{H}$ MR spectroscopy of thyroid carcinoma. Eur J Radiol 2005;54:112-17

12. Maheshwari SR, Mukherji SK, Neelon B, et al. The choline/creatine ratio in five benign neoplasms: comparison with squamous cell carcinoma by use of in vitro MR spectroscopy. AJNR Am J Neuroradiol 2000;21:1930-35

13. Krouwer HG, Kim TA, Rand SD, et al. Single-voxel proton MR spectroscopy of nonneoplastic brain lesions suggestive of a neoplasm. AJNR Am J Neuroradiol 1998;19:1695-703

14. Rand SD, Prost R, Haughton V, et al. Accuracy of single-voxel proton MR 
spectroscopy in distinguishing neoplastic from nonneoplastic brain lesions. AJNR Am J Neuroradiol 1997;18:1695-704

15. King AD, Yeung DK, Ahuja AT, et al. Salivary gland tumors at in vivo proton MR spectroscopy. Radiology 2005;237:563-69

16. King AD, Yeung DK, Yu KH, et al. Monitoring of treatment response after chemoradiotherapy for head and neck cancer using in vivo $1 \mathrm{H}$ MR spectroscopy. Eur Radiol 2010;20:165-72. Epub 2009 Aug 5

17. Bezabeh T, Odlum $\mathrm{O}$, Nason $\mathrm{R}$, et al. Prediction of treatment response in head and neck cancer by magnetic resonance spectroscopy. AJNR Am J Neuroradiol 2005;26:2108-13

18. King $\mathrm{AD}$, Yeung $\mathrm{DK}, \mathrm{Yu} \mathrm{KH}$, et al. Pretreatment and early intratreatment prediction of clinicopathologic response of head and neck cancer to chemoradiotherapy using 1H-MRS. J Magn Reson Imaging 2010;32:199-203

19. Lee TY. Functional CT: physiological models. Trends Biotechnol 2002;20:S3-10

20. Lee TY, Purdie TG, Stewart E. CT imaging of angiogenesis. Q J Nucl Med 2003;47:171-87

21. Gandhi D, Hoeffner EG, Carlos RC, et al. Computed tomography perfusion of squamous cell carcinoma of the upper aerodigestive tract: initial results. J Comput Assist Tomogr 2003;27:687-93

22. Rumboldt Z, Al-Okaili R, Deveikis JP. Perfusion CT for head and neck tumors: pilot study. AJNR Am J Neuroradiol 2005;26:1178-85

23. Bisdas S, Baghi M, Smolarz A, et al. Quantitative measurements of perfusion and permeability of oropharyngeal and oral cavity cancer, recurrent disease, and associated lymph nodes using first-pass contrast-enhanced computed tomography studies. Invest Radiol 2007;42:172-79

24. Bisdas S, Rumboldt Z, Surlan-Popovic K, et al. Perfusion CT in squamous cell carcinoma of the upper aerodigestive tract: long-term predictive value of baseline perfusion CT measurements. AJNR Am J Neuroradiol 2010;31:57681. Epub 2009 Oct 29

25. Bisdas S, Nguyen SA, Anand SK, et al. Outcome prediction after surgery and chemoradiation of squamous cell carcinoma in the oral cavity, oropharynx, and hypopharynx: use of baseline perfusion CT microcirculatory parameters vs. tumor volume. Int J Radiat Oncol Biol Phys 2009;73:1313-18

26. Zima A, Carlos R, Gandhi D, et al. Can pretreatment CT perfusion predict response of advanced squamous cell carcinoma of the upper aerodigestive tract treated with induction chemotherapy? AJNR Am J Neuroradiol 2007;28:328-34

27. Surlan-Popovic K, Bisdas S, Rumboldt Z, et al. Changes in perfusion CT of advanced squamous cell carcinoma of the head and neck treated during the course of concomitant chemoradiotherapy. AJNR Am J Neuroradiol 2010;31: 570-75. Epub 2009 Oct 29

28. Ash L, Teknos TN, Gandhi D, et al. Head and neck squamous cell carcinoma: CT perfusion can help noninvasively predict intratumoral microvessel density. Radiology 2009;251:422-28

29. Lacerda S, Law M. Magnetic resonance perfusion and permeability imaging in brain tumors. Neuroimaging Clin N Am 2009;19:527-57

30. Ludemann L, Warmuth C, Plotkin M, et al. Brain tumor perfusion: comparison of dynamic contrast enhanced magnetic resonance imaging using $\mathrm{T} 1, \mathrm{~T} 2$, and $\mathrm{T} 2{ }^{*}$ contrast, pulsed arterial spin labeling, and $\mathrm{H} 2(15) \mathrm{O}$ positron emission tomography. Eur J Radiol 2009;70:465-74

31. Bisdas S, Baghi M, Wagenblast J, et al. Gadolinium-enhanced echo-planar T2weighted MRI of tumors in the extracranial head and neck: feasibility study and preliminary results using a distributed-parameter tracer kinetic analysis. J Magn Reson Imaging 2008;27:963-69

32. Cao Y, Popovtzer A, Li D, et al. Early prediction of outcome in advanced headand-neck cancer based on tumor blood volume alterations during therapy: a prospective study. Int J Radiat Oncol Biol Phys 2008;72:1287-90

33. de Bondt RB, Hoeberigs MC, Nelemans PJ, et al. Diagnostic accuracy and additional value of diffusion-weighted imaging for discrimination of malignant cervical lymph nodes in head and neck squamous cell carcinoma. Neuroradiology 2009;51:183-92

34. Eida S, Sumi M, Sakihama N, et al. Apparent diffusion coefficient mapping of salivary gland tumors: prediction of the benignancy and malignancy. AJNR Am J Neuroradiol 2007;28:116-21

35. Holzapfel K, Duetsch S, Fauser C, et al. Value of diffusion-weighted MR imaging in the differentiation between benign and malignant cervical lymph nodes. Eur J Radiol 2009;72:381-87

36. Srinivasan A, Dvorak R, Perni K, et al. Differentiation of benign and malignant pathology in the head and neck using $3 \mathrm{~T}$ apparent diffusion coefficient values: early experience. AJNR Am J Neuroradiol 2008;29:40-44

37. Srinivasan A, Dvorak R, Rohrer S, et al. Initial experience of 3-Tesla apparent diffusion coefficient values in characterizing squamous cell carcinomas of the head and neck. Acta Radiol 2008;49:1079-84

38. Vandecaveye V, De Keyzer F, Vander Poorten V, et al. Head and neck squamous cell carcinoma: value of diffusion-weighted MR imaging for nodal staging. Radiology 2009;251:134-46

39. Habermann CR, Arndt C, Graessner J, et al. Diffusion-weighted echo-planar MR imaging of primary parotid gland tumors: is a prediction of different histologic subtypes possible? AJNR Am J Neuroradiol 2009;30:591-96

40. Habermann CR, Gossrau P, Graessner J, et al. Diffusion-weighted echo-planar
MRI: a valuable tool for differentiating primary parotid gland tumors? Rofo 2005; 177:940-45

41. Matsushima N, Maeda M, Takamura M, et al. Apparent diffusion coefficients of benign and malignant salivary gland tumors: comparison to histopathological findings. J Neuroradiol 2007;34:183-89

42. Dirix P, De Keyzer F, Vandecaveye V, et al. Diffusion-weighted magnetic resonance imaging to evaluate major salivary gland function before and after radiotherapy. Int J Radiat Oncol Biol Phys 2008;71:1365-71

43. Vandecaveye V, De Keyzer F, Nuyts S, et al. Detection of head and neck squamous cell carcinoma with diffusion weighted MRI after (chemo)radiotherapy: correlation between radiologic and histopathologic findings. Int J Radiat Oncol Biol Phys 2007;67:960-71

44. Abdel Razek AA, Kandeel AY, Soliman N, et al. Role of diffusion-weighted echoplanar MR imaging in differentiation of residual or recurrent head and neck tumors and post-treatment changes. AJNR Am J Neuroradiol 2007;28:1146-52

45. Vandecaveye V, de Keyzer F, Vander Poorten V, et al. Evaluation of the larynx for tumour recurrence by diffusion-weighted MRI after radiotherapy: initial experience in four cases. Br J Radiol 2006;79:681-87

46. Hamstra DA, Lee KC, Moffat BA, et al. Diffusion magnetic resonance imaging an imaging treatment response biomarker to chemoradiotherapy in a mouse model of squamous cell cancer of the head and neck. Transl Oncol 2008;1:187-94

47. Galban CJ, Mukherji SK, Chenevert TL, et al. A feasibility study of parametric response map analysis of diffusion-weighted magnetic resonance imaging scans of head and neck cancer patients for providing early detection of therapeutic efficacy. Transl Oncol 2009;2:184-90

48. Kim S, Loevner L, Quon H, et al. Diffusion-weighted magnetic resonance imaging for predicting and detecting early response to chemoradiation therapy of squamous cell carcinomas of the head and neck. Clin Cancer Res 2009;15:986-94

49. Kato $\mathrm{H}$, Kanematsu $\mathrm{M}$, Tanaka $\mathrm{O}$, et al. Head and neck squamous cell carcinoma: usefulness of diffusion-weighted MR imaging in the prediction of a neoadjuvant therapeutic effect. Eur Radiol 2009;19:103-09

50. Vandecaveye V, Dirix P, De Keyzer F, et al. Predictive value of diffusionweighted magnetic resonance imaging during chemoradiotherapy for head and neck squamous cell carcinoma. Eur Radiol 2010;20:1703-14

51. Fukui MB, Blodgett TM, Snyderman CH, et al. Combined PET-CT in the head and neck. Part 2. Diagnostic uses and pitfalls of oncologic imaging. Radiographics 2005;25:913-30

52. Wong RJ, Lin DT, Schoder H, et al. Diagnostic and prognostic value of [F18] fluorodeoxyglucose positron emission tomography for recurrent head and neck squamous cell carcinoma. J Clin Oncol 2002;20:4199-208

53. Al-Ibraheem A, Buck A, Krause BJ, et al. Clinical applications of FDG PET and PET/CT in head and neck cancer. J Oncol 2009;2009:208725. Epub 2009 Aug 20

54. Ong SC, Schoder H, Lee NY, et al. Clinical utility of 18F-FDG PET/CT in assessing the neck after concurrent chemoradiotherapy for locoregional advanced head and neck cancer. J Nucl Med 2008;49:532-40

55. Isles MG, McConkey C, Mehanna HM. A systematic review and meta-analysis of the role of positron emission tomography in the follow up of head and neck squamous cell carcinoma following radiotherapy or chemoradiotherapy. Clin Otolaryngol 2008;33:210-22

56. Kao J, Vu HL, Genden EM, et al. The diagnostic and prognostic utility of positron emission tomography/computed tomography-based follow-up after radiotherapy for head and neck cancer. Cancer 2009;115:4586-94

57. Subramaniam RM, Truong M, Peller P, et al. Fluorodeoxyglucose-positronemission tomography imaging of head and neck squamous cell cancer. AJNR Am J Neuroradiol 2010;31:598-604. Epub 2009 Nov 12

58. Troost EG, Schinagl DA, Bussink J, et al. Innovations in radiotherapy planning of head and neck cancers: role of PET. J Nucl Med 2010;51:66-76. Epub 2009 Dec 15

59. Akter M, Hirai T, Minoda R, et al. Diffusion tensor tractography in the headand-neck region using a clinical 3-T MR scanner. Acad Radiol 2009;16:858-65

60. Schoder H, Fury M, Lee N, Kraus D. PET monitoring of therapy response in head and neck squamous cell carcinoma. J Nucl Med 2009;50(suppl 1):74S-88S

61. Eschmann SM, Paulsen F, Reimold M, et al. Prognostic impact of hypoxia imaging with $18 \mathrm{~F}$-misonidazole PET in non-small cell lung cancer and head and neck cancer before radiotherapy. J Nucl Med 2005;46:253-60

62. Rischin D, Hicks RJ, Fisher R, et al. Prognostic significance of [18F]-misonidazole positron emission tomography-detected tumor hypoxia in patients with advanced head and neck cancer randomly assigned to chemoradiation with or without tirapazamine: a substudy of Trans-Tasman Radiation Oncology Group Study 98.02. J Clin Oncol 2006;24:2098-104

63. Kim JJ, Tannock IF. Repopulation of cancer cells during therapy: an important cause of treatment failure. Nat Rev Cancer 2005;5:516-25

64. Shields AF, Grierson JR, Dohmen BM, et al. Imaging proliferation in vivo with [F-18]FLT and positron emission tomography. Nat Med 1998;4:1334-36

65. Cobben DC, van der Laan BF, Maas B, et al. 18F-FLT PET for visualization of laryngeal cancer: comparison with 18F-FDG PET. J Nucl Med 2004;45:226-31

66. Linecker A, Kermer C, Sulzbacher I, et al. Uptake of (18)F-FLT and (18)F-FDG in primary head and neck cancer correlates with survival. Nuklearmedizin 2008;47:80-85, quiz N12

67. Czernin J, Benz MR, Allen-Auerbach MS. PET/CT imaging: the incremental value of assessing the glucose metabolic phenotype and the structure of cancers in a single examination. Eur J Radiol 2010;73:470-80. Epub 2010 Jan 25 